\title{
Renal primitive neuroectodermal tumour in childhood: Case report and review of literature
}

\author{
Maeed Asiri, MD; Ahmed Al-Sayyad, MD, FRCSC
}

\begin{abstract}
Primitive neuroectodermal tumour (PNET) is presumed to be of neural crest origin, mostly presenting as bone or soft tissue masses. It usually occurs in the trunk or axial skeleton; while renal PNET is considered an extremely rare tumour. We report a case of 11-yearold male who presented with right flank pain and gross hematuria after suffering blunt trauma. During investigations, he was found to have a large renal mass on computed tomography. He underwent a right radical nephrectomy where the pathology report showed PNET of the kidney. The patient received chemotherapy afterwards. Despite the chemotherapy, he had a local tumour recurrence 3 months after and continued to deteriorate and developed distant metastasis. Primitive neuroectodermal tumour of the kidney is a distinct and rare entity. It is very aggressive, with a poor survival despite combined modality treatment.
\end{abstract}

Can Urol Assoc J 2010;4(6):E158-E160

\section{Case report}

A previously healthy 11 -year-old boy came to the emergency department complaining of right flank pain and gross hematuria for 1 week duration after suffering blunt trauma. He had no other associated symptoms. Physical examination revealed a palpable firm and large right upper quadrant mass. His hemoglobin and serum creatinine levels were normal.

A computerized tomography (CT) scan of the abdomen demonstrated a large heterogeneous enhancing mass with areas of cystic degeneration noted involving the upper and mid regions of the right kidney. It measured $10 \times 9 \times 11 \mathrm{~cm}$ with areas of necrosis and hemorrhage and no lymphadenopathy (Fig. 1). Although necrosis and hemorrhage are pathological diagnosis, areas of high attenuation in pre-contrast CT might suggest hemorrhage, while after intravenous contrast non-enhanced areas may suggest necrosis. There was no evidence of renal vein or vena caval extension by magnetic resonance imaging (Fig. 2).
Chest $\mathrm{CT}$ and radionuclide bone scan showed no evidence of a metastatic disease. The patient was referred to the physicians in the pediatric oncology department and they agreed with our plan of performing a radical nephrectomy first. The patient then underwent a right radical nephrectomy and hilar lymph node dissection. There was only one grossly enlarged lymph node that was resected along with other areas of hilar fat. Grossly, the kidney weighted $700 \mathrm{~g}$ and measured $16 \times 14 \times 9 \mathrm{~cm}$. On sectioning, there was a huge mass almost replacing the whole kidney, sparing only a rim of the kidney at the lower pole. Margins were negative and adrenal gland was unremarkable.

Microscopically, sections revealed neoplastic growth displayed in vaguely nodular pattern with intersecting thick fibrous septea. The neoplastic growth is composed of sheets of undifferentiated small blue cells with occasional HomerWright rosettes. These cells have basophilic chromatin and frequent mitosis. Also there are areas of spindle fibroblastlike cell proliferation. No glycogen globules were identified. There was marked hemorrhage and occasional necrotic foci encountered within the tumour. Vascular and perineural invasion were seen. One hilar lymph node showed reactive changes and was negative for malignancy. The adrenal gland was normal.

A panel of immunohistochemical markers was performed which included meuron-specific enolase (NSE), synaptophysin, macrophage inhibitory cytokine (MIC-2), Wilms' tumour and P53.The tumour cells were positive for MIC2, NSE and synaptophysin, while they were negative for the rest of the markers.

The patient was seen by the pediatric oncology team before discharge and scheduled to receive multi-agent chemotherapy. During his 3-month follow-up, he complained of abdominal pain and lower limb swelling. A CT scan was repeated and revealed a local tumour recurrence. Although a biopsy of the mass was not taken, he continued to receive chemotherapy. Despite this, he deteriorated and developed distant metastasis. 


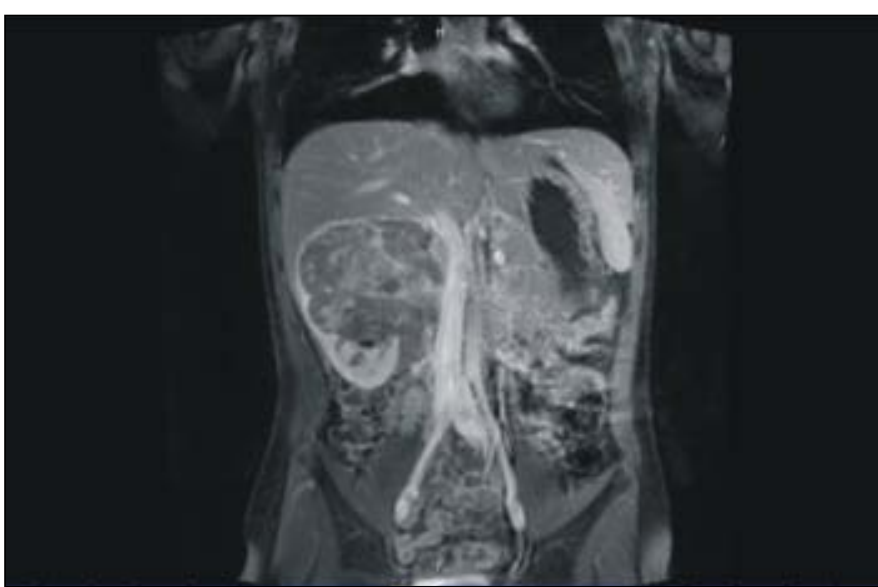

Fig. 1. A computed tomography scan of the abdomen and pelvis showing large, heterogeneous and enhancing right renal mass

\section{Discussion}

Primitive neuroectodermal tumour is a malignant small cell neoplasm of neural crest origin. It was first described by Arthur Pourdy Stout in 1918, where it occurred in the ulnar nerve. ${ }^{1}$ It is a very rare tumour representing about $1 \%$ of all sarcomas. ${ }^{2}$ It can occur in the trunk, extremities, brain, spinal cord and sympathetic nervous system, as well as peripheral tissues, like the chest wall (Askin's tumour), ${ }^{3}$ paraspinal region and, less commonly, the genitourinary tract. ${ }^{4}$ In 1921 , Ewing described this tumour in the bone, which became known as Ewing's sarcoma. ${ }^{5}$ Renal PNET was first reported by Mor and colleagues. ${ }^{6}$ There are only 4 reported series of renal PNET, which includes articles by Parham and colleagues, ${ }^{7}$ Jimenez and colleagues, ${ }^{8}$ Carlos and colleagues ${ }^{9}$ and Yuvaraja and colleagues. ${ }^{10}$

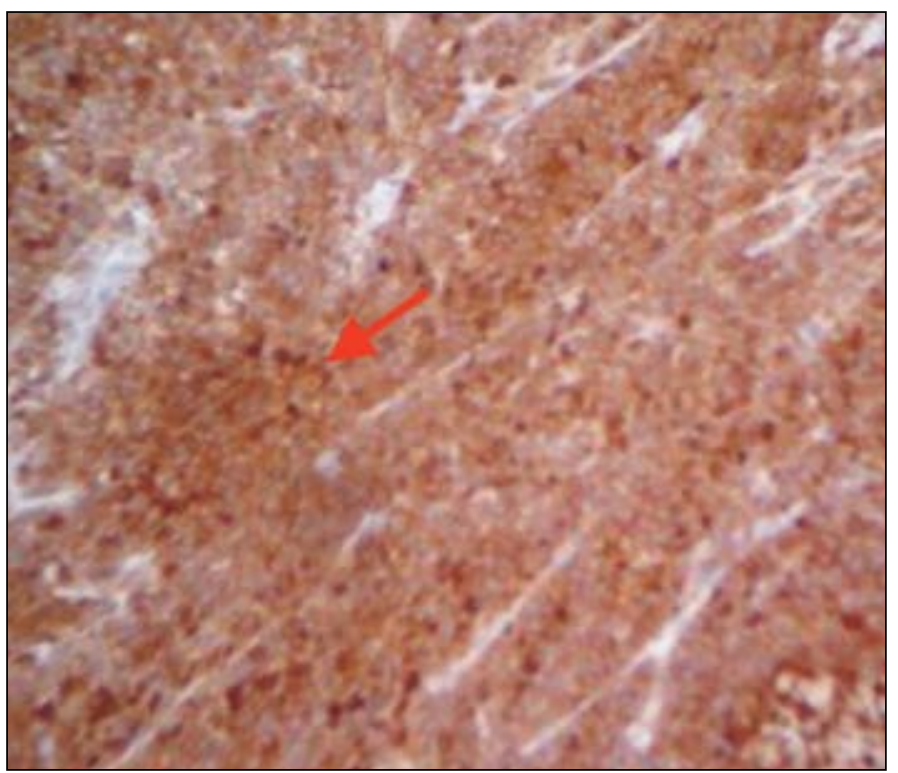

Fig. 3. Small blue cells with occasional Homer-Wright rosettes.

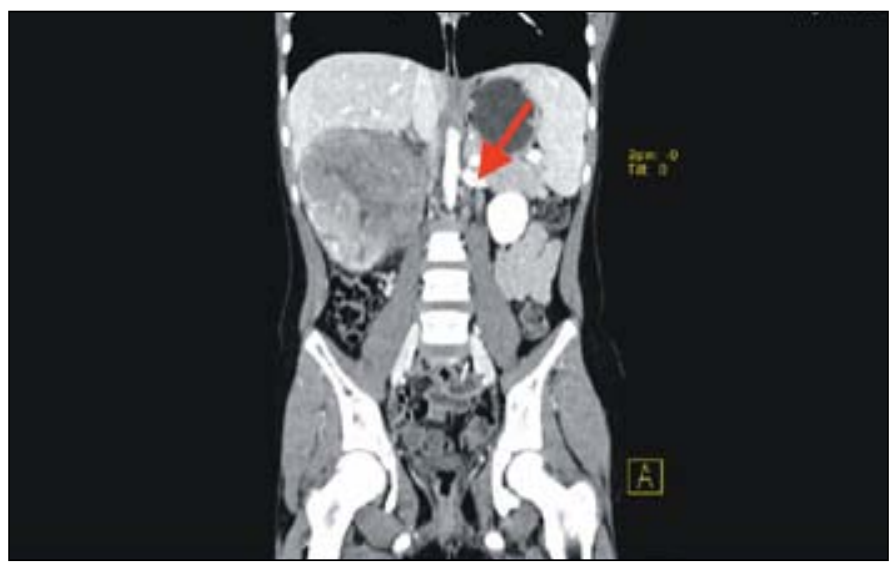

Fig. 2. A magnetic resonance imaging of the abdomen and pelvis, which shows the patent's inferior vena cava with no evidence of thrombosis.

Renal PNET is extremely rare with fewer than 50 cases in the English literature. Renal PNET is a highly aggressive malignant neoplasm and is more aggressive than PNET arising from other sites. ${ }^{11}$

PNET also occurs in children and adolescents. The age range for this condition is from 4 to 61 years. ${ }^{7}$ To our knowledge, our case is the fourth reported case in the pediatric age group; most of the reported cases occur with patients in their second or third decades of life. ${ }^{12,13}$ The male to female ratio is about $3: 1$. The presenting symptoms and clinical signs are non-specific and similar to those of other renal tumours. ${ }^{14-18}$

The diagnosis of renal PNET is made usually postoperatively, based on histopathology and the panel of immunohistochemical stains. ${ }^{19}$ Differential diagnosis includes

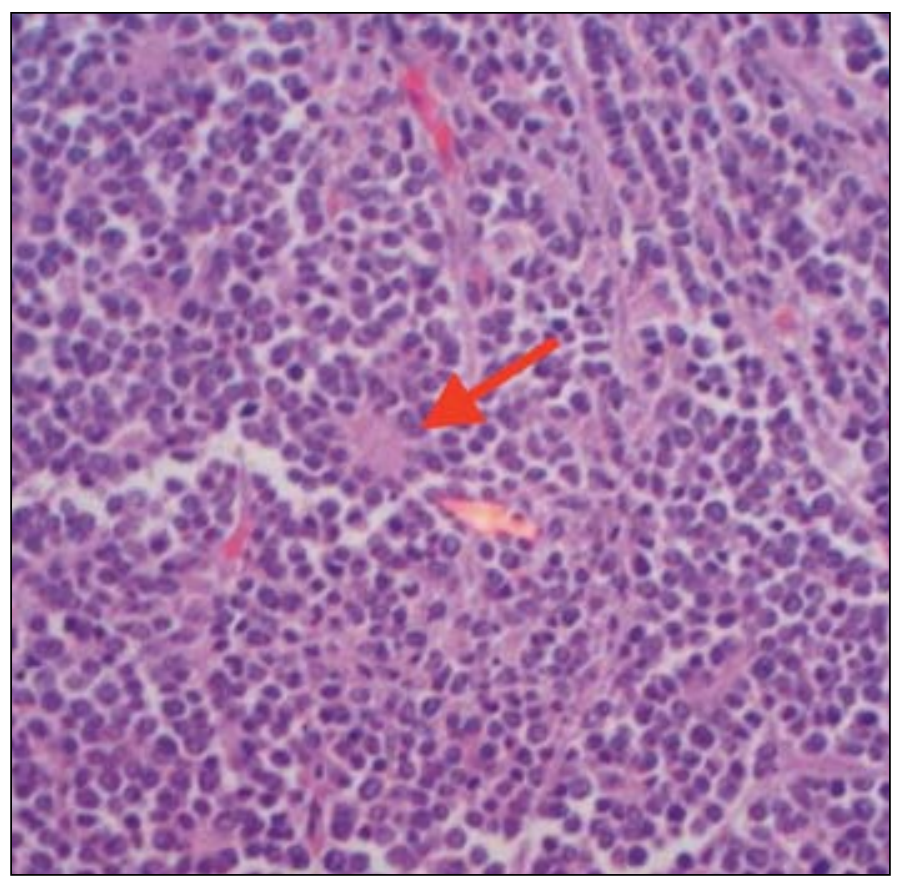

Fig. 4. Tumour cells were positive for immunohistochemical marker MIC2. 
Wilms' tumour, neuroblastoma, clear cell carcinoma and lymphoma. Radiographic features of renal PNET are nonspecific and are usually presented as a huge renal mass with or without calcification. ${ }^{20}$ Histologically, PNET consists of sheets and nests of primitive small round blue cells and presence of Homer-Wright rosettes (Fig. 3, Fig. 4). ${ }^{21}$ Immunohistochemical staining is crucial in the diagnosis. Renal PNET is positive for MIC2 in more than $90 \%$ to $95 \%$ of the cases. Other helpful markers are neuron-specific enolase and vimentin, synaptophysin and S100 protein..$^{20-25}$

Furthermore, cytogenetic studies showed that renal PNET is consistently associated with translocation of the long arms of chromosome 11 and 22, $\mathrm{t}(11: 22)$ ( $q 24: q 12)$ in more than $90 \%$ of the tumours; it is also characteristic of PNET and Ewing's sarcoma. Fluorescence in-situ hybridization (FISH) allows confirmation of the PNET/Ewing sarcoma specific $t(11: 22)$ and $t(21: 22) .{ }^{26}$ Unfortunately, cytogenetic studies was not performed in our case because it is not available. ${ }^{24}$

Renal PNET is a highly aggressive tumour and almost $50 \%$ of patient present with distant metastases, most commonly to regional lymph nodes, lungs and liver. It has a high tendency toward early local recurrence after treatment. The survival rate is poor and most patients die within 1 year of the diagnosis; although, in some of the previous reports, the 5 -year disease-free survival rate was $45 \%$ to $55 \%{ }^{2-18}$

Since PNET biologically behaves like Ewing's sarcoma, it should be treated with a combination of radical nephrectomy and chemotherapy, which includes vincristine, doxorubicin, cyclophosphamide, etoposide and ifosfamide. ${ }^{27}$ In cases where there is an incomplete resection or positive margin or recurrence of the tumour, radiation is recommended. However, further studies are needed to find the appropriate treatment protocol.

\section{Conclusion}

Primitive neuroectodermal tumour of the kidney is a distinct entity. It is very aggressive with a poor survival, despite combined modality treatment. Although PNET is rare, we should be aware of its presence. Cytogenetic studies and immunohistochemistry for CD99 are very helpful in the diagnosis of renal PNET.

Department of Urology, King Abdul Aziz University Hospital, Jeddah, Kingdom of Saudi Arabia

Competing interests: None declared.

This paper has been peer-reviewed.

\section{References}

1. Sout AP. A tumour of the unar nerve. Proc N Y Pathol Sco 1918;18:2-12.

2. Enzinger FM, Weiss SW. Primitive neuroectodermal tumours and related lesions. In: Weiss SW, Enzinger FM (editors). Soft tissue tumours. 3rd edition. St. Louis, MO: Mosby; 1995:929-64.

3. Indada K, Nakahara K, Fujii Y, et al. A case of primitive neuroectodermal tumour of the chest wall (Askin's tumour) J Jpn Assoc Thoracic Surg 1994;42;254-8.

4. Gupta NP, Singh BP, Raina V, et al. Primitive neuroectodermal kidney tumour: 2 case reports and review of the literature. J Urol 1995; 153:1890-2.

5. Ewing J. Diffuse endothelioma of bone. Proc N Y Pathol Soc 1921;21:17-24.

6. Mor Y, Nass D, Raviv G, et al. Malignant peripheral primitive neuroectodermal tumour (PNET) of the kidney. Med Pediatr Oncol 1994;23:437-40.

7. Parham D, Roloson G, Feely M, et al. Primary malignant neuroepithelial tumours of the kidney. Am J Surg Pathol 2001;25:133-46.

8. Jimenez R, Folpe A, Lapham R , et al. Primary Ewing's sarcoma/primitive neuroectodermal tumour of the kidney. Am J Surg Pathol 2002;26:320-7.

9. Carlos R, Neyssa M, Barry D, et al. Is primitive neuroectodermal tumour of the kidney a distinct entity? Am Canc Soc 1997;79:2243-50

10. Yuvaraja $B$, Thyavihally, Hemant $B$, et al. Primitive neuroectodermal tumour of the kidney: A single institute series of 16 patients. Urology 2008;71:292-6.

11. Lam JS, Hensle TW, Delbelenko $L$, et al. Organ confined primitive neuroectodermal tumour arising from the kidney. J Pediatr Surg 2003;38:619-21.

12. Badar Q, Ali N, Abbasi N, et al. Ewing's sarcoma/PNET of kidney in 13-year-old girl. J Pak Med Assoc 2010;60:314-5.

13. Citak EC, Oguz A, Karadeniz C, et al. Primitive euroectodermal tumor of the kidney in a child. Pediatr Hematol Oncol 2009;26:481-6.

14. Fellinger EJ, Garin-Chesa P, Glasser DB, et al. Comparison of cell surface antigen HBA71 (p30/32MIC2), neuron-specific enolase and vimentin in the immunohistochemical analysis of Ewings sarcoma of bone. Am J Surg Pathol 1992;16:746-55.

15. Mentzel T, Bultitude MI, Fletcher CD. Primary primitive neuroecto-dermal tumour of the kidney in an adult: clinico-pathologic and immunohistochemical case report. Pathologe 1994;15:124-8.

16. Quezado M, Benjamin DR, Tsokos M. EWS/FLl-1 fusion transcripts in three peripheral primitive neuroectodermal tumours of the kidney. Hum Pathol 1997;28:767-71.

17. Chan YF, Llewellyn H. Intrarenal primitive neuroectodermal tumour. Br J Urol 1994;73:326-7.

18. Furman J, Murphy WM, Jelsma PF, et al. Primary primitive neuroectodermal tumour of the kidney: case report and review of the literature. Am I Clin Pathol 1996;106:339-44.

19. Sternberg S. Diagnostic Surgical pathology. 3rd edition. Baltimore, MD: Lippincott Williams \& Wilkins; 1999:1809-10.

20. Maccioni F, Della Rocca C, Salvi PF, et al. Malignant peripheral neuroectodermal tumour (MPNET) of the kidney. Abdom Imag 2000;25:103-6.

21. Romero FR, Rais-Bahrami S, Muntener M, et al. Metastatic primitive neuroectoderm tumour of the kidney. Urol Int 2007;78:268-88

22. Marley EF, Liapis H, Humphrey PA, et al. Primitive neuroectodermal tumour of the kidney: another enigma. A pathologic, immunohistochemical and molecular diagnostic study. Am I Surg Pathol 1997;21:354-9.

23. Weiss SW, Goldblum JR. Primitive neuroectodermal tumors and related lesions. In: Weiss SW, Enzinger FM (editors). Soft tissue tumours. 3rd edition. St. Louis, MO: Mosby; 2002:1265-321.

24. Perlman EJ, Dickman PS, Askin FB, et al. Ewings sarcoma: routine diagnostic utilization of MIC2 analysis. A Pediatric Oncology Group/Childrens Cancer Group Intergroup study. Hum Pathol 1994;25:304-7.

25. Sheaff M, McManus A, Scheimberg I, et al. Primitive neuroectodermal tumour of the kidney confirmed by fluores-cence in situ hybridization. Am I Surg Pathol 1997;21:461-8.

26. Takeuchi T, Iwasaki H, Ohijmi Y, et al. Renal primitive neuroecto-dermal tumour: An immunohistochemical and cytogenetic analysis. Pathol Int 1996;46:292-7.

27. Granowetter L. Ewing's sarcoma and extracranial primitive neiroectodermal tumour. Curr Opin Oncol 1996;8:305-10.

Correspondence: Dr. Ahmed Al-Sayyad, Assistant Professor, Department of Urology, King Abdul Aziz University, Jeddah, Saudi Arabia University; aboassil@yahoo.com 